(C) Masson, Paris, 1980

\title{
Deux Filaires du genre Eufilaria chez le Merle: développement chez Culicoides nubeculosus (*)
}

\author{
par O. BAIN \\ Laboratoire de Zoologie (Vers), associé au C.N.R.S., Muséum national d'Histoire naturelle, \\ 43, rue Cuvier, $F 75231$ Paris Cedex 05.
}

RESUME. Turdus merula à Nevers (France) présente deux espèces de microfilaires qui évoluent toutes deux en huit jours à $26^{\circ} \mathrm{C}$ chez Culicoides nubeculosus. Nous déterminons comme Eufilaria delicata Supperer, 1958, les femelles ayant les plus grandes microfilaires, le mâle à spicules peu inégaux et le stade infestant le plus long. Nous rattachons à l'espèce à microfilaires plus courtes les mâles à spicules plus inégaux et le stade infestant le plus court et la nommons $E$. bartlettae n. sp.; la femelle reste inconnue. La détermination des $\mathrm{L}_{I I}$ reste provisoire puisqu'elle n'est fondée que sur la notion des fréquences relatives des deux espèces.

Les stades infestants actuellement décrits chez les Onchocercidae d'Oiseaux évoluant chez les Culicoides et les Simulies (genre Splendidofilaria, Chandlerella et Eufilaria) sont proches les uns des autres, mais les extrémités caudales des premier et troisième stades diffèrent d'un genre à l'autre.

Two Filariae of the genus Eufilaria in Turdus merula; development in Culicoides nubeculosus.

SUMMARY. Turdus merula from Nevers (France) is infected by two species of microfilariae which both evolve in eight days $\left(26^{\circ} \mathrm{C}\right)$ in Culicoides nubeculosus. We determine as Eufilaria delicata Supperer, 1958, the fernales with the longest microfilariae, the male with slightly unequal spicules and the largest infective larvae. We consider the species with the shorter microfilariae, males with more markedly unequal spicules

(*) Ce travail a été effectué grâce à une subvention de l'Organisation Mondiale de la Santé. Accepté le $1^{\text {er }}$ mars 1980. 
and a relatively short infective larva as $\mathrm{E}$. bartlettae $n$. sp. The female of this species is unknown. The identification of the infective larvae is provisional since it is based on the relative frequency of the two species.

The infective larvae of Onchocercidae in birds which have been described as developing in Culicoides and simulids (genera Splendidofilaria, Chandlerella and Eufilaria) are morphological similar but the caudal extramities of the first and third stages differ between the genera.

\section{Introduction}

Deux merles (Turdus merula), capturés les 15 et 16 novembre 1979 à la Station ornithologique du Bec d'Allier $\left(^{*}\right)$ (Val-de-Loire) ont des microfilaires sanguines qui paraissent se rapporter au genre Eufilaria (corps peu allongé, tête plate, queue pointue, pas de gaine).

Le merle mâle 104 EJ, à microfilarémie particulièrement basse (les microfilaires n'ont pas été mesurées), est autopsié le 3-12-1979 : quatre mâles d'Eufilaria sont récoltés dans le tissu conjonctif qui entoure l'œsophage et la trachée.

Le merle femelle $105 \mathrm{EJ}$ a une microfilarémie qui atteint la nuit 15-22 microfilaires pour $10 \mu \mathrm{l}$. Deux types de microfilaires coexistent; les unes longues de 150 $170 \mu \mathrm{m}$, les autres de $110-122 \mu \mathrm{m}$; ces dernières sont 5 à 10 fois plus nombreuses. Ce merle est utilisé pour tenter de réaliser les cycles chez des Culicoides nubeculosus d'élevage. Le développement des deux microfilaires s'effectuent en 8 jours à $26^{\circ}$.

A l'autopsie de ce merle, effectuée le 17-12-1979, le sang cardiaque se révèle extrêmement riche en microfilaires; les deux espèces coexistent avec la même abondance relative que dans le sang périphérique. Un mâle et quatre femelles d'Eufilaria sont récoltés dans le tissu conjonctif péri-œsophagien. Les femelles ont toutes des microfilaires longues; leurs dimensions, celles des microfilaires et celles du mâle (particulièrement les spicules peu inégaux) permettent d'identifier ce matériel à $E$. delicata Supperer, 1958.

Les quatre mâles $104 \mathrm{EJ}$ ont une morphologie distincte: les spicules gauche et droit sont plus inégaux; ces mâles, que nous rattachons par élimination à la petite microfilaire, constituent une nouvelle espèce, E. bartlettae n. sp.

Les deux types morphologiques trouvés chez les larves récoltées chez $C$. nubeculosus ont la même fréquence relative (1 à 5) que les deux microfilaires rencontrées dans le sang de l'oiseau donneur. Nous désignons donc sous le nom de E. bartlettae n. sp. l'espèce fréquente et de E. delicata l'espèce plus rare, mais cette attribution des formes larvaires est provisoire puisqu'elle ne repose que sur une simple probabilité.

(*) Nous remercions vivement $\mathrm{M}$. Clavier, directeur de la station, qui nous a aidé efficacement. 


\section{I - Eufilaria delicata}

MATÉRIEL : 4 femelles, 1 mâle et une moitié postérieure de femelle ; lot $105 \mathrm{EJ}$.

La morphologie est représentée sur la figure 1 et $2 \mathrm{~A}$ à $F$.

Dimensions : femelles : corps longs de 23,3,19,1, 24,9 et 20,4 mm, larges de 130, 130, 120 et $90 \mu \mathrm{m}$; anneau nerveux à $170,150,185$ et $220 \mu \mathrm{m}$ de l'apex ; vulve à 645,534 , 740 et $800 \mu \mathrm{m}$ de l'apex; queue longue de 55, 100, 55 et $78 \mu \mathrm{m}$; ovéjecteur, mesuré
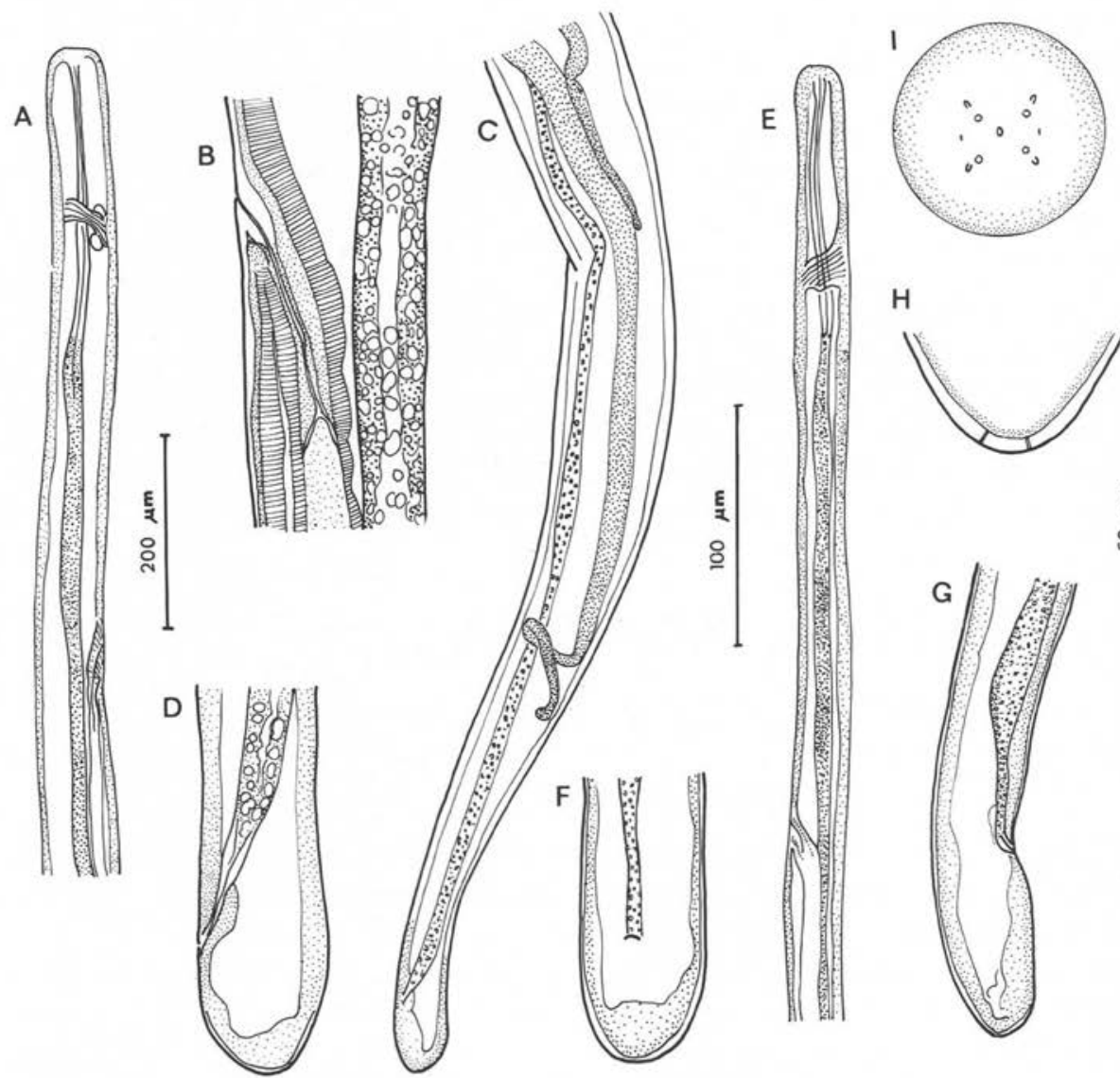

$\mathrm{H}$
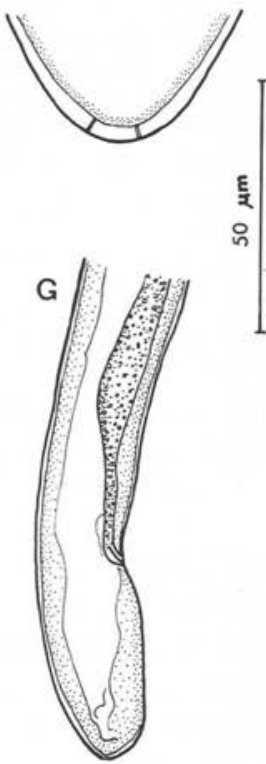

Fig. 1. Eufilaria delicata, femelle; A : région antérieure, vue latérale; B : région distale de, l'ovéjecteur et portion d'intestin; C: région postérieure, vue latérale; D et F: queue, vues latérale et ventrale; $\mathrm{E}$, autre femelle: région antérieure, vue latérale; G : idem, région caudale, vue latérale; $\mathrm{H}$ : extrémité caudale, vue ventrale; $\mathrm{I}$ : tête, vue apicale.

(A, E, C, éch. $200 \mu \mathrm{m}$; D, F, G, éch. $100 \mu \mathrm{m}$; B, H, I, éch. $50 \mu \mathrm{m}$ ). 
sur une femelle, long de $2025 \mu \mathrm{m}$; ovaire le plus postérieur situé de 450 à $1670 \mu \mathrm{m}$ de l'extrémité postérieure ; l'autre ovaire de 400 à $2570 \mu \mathrm{m}$ de cette extrémité.

Mâle : corps long de 10,85 mm, large de $65 \mu \mathrm{m}$; anneau nerveux de $182 \mu \mathrm{m}$ de l'apex ; œsophage long de $260 \mu \mathrm{m}$; queue, spicule gauche et spicule droit longs de $20 \mu \mathrm{m}$, $58 \mu \mathrm{m}$ et $50 \mu \mathrm{m}$; testicule à $325 \mu \mathrm{m}$ de l'apex.

Microfilaire :

- dans le sang, immobilisées par la chaleur : corps longs de 146 à $170 \mu \mathrm{m}(170,158$, $155,146,150$ ) et larges de 4,5 à $5 \mu \mathrm{m}$;

- dans l'ovéjecteur des femelles fixées à l'alcool chaud : 107 à $155 \mu \mathrm{m}(133,142,136$, $140,142,155,155,145,107,130,140,135$ et 142$)$;

- 2 microfilaires sanguines, colorées vitalement : corps longs de $170 \mu \mathrm{m}$ et $158 \mu \mathrm{m}$, larges de 4,5 et $5 \mu \mathrm{m}$; pore excréteur à ? et $48 \mu \mathrm{m}$ et noyau excréteur à 63 et $55 \mu \mathrm{m}$ de l'apex; corps interne à 87 et $78 \mu \mathrm{m}$ de l'apex et long de 16 et $17 \mu \mathrm{m} ; \mathrm{R}_{1}$ à 115 et $108 \mu \mathrm{m}$ de l'apex. Queue longue de ? et $33 \mu \mathrm{m}$ (fig. $3 \mathrm{~A}$ ).

\section{Discussion.}

Le genre Eufilaria Seurat, 1921, auquel appartiennent ces Filaires, comprend 10 espèces (Bartlett, 1979) dont une, E. delicata Supperer, 1958, est parasite de divers Turdus et de Garrulus glandarius en Autriche.

Les dimensions générales de nos spécimens, la taille peu inégale des spicules du mâle ( $8 \mu$ d'écart), la taille de la microfilaire et de son corps interne, sont semblables à celles d'E. delicata et nous identifions notre matériel à cette espèce.

\section{II - Eufilaria bartlettae n. sp.}

MATÉRIEL : 4 mâles 104 EJ, dont l'holotype ; à ce matériel, nous rattachons la microfilaire sanguine de petite taille.

La morphologie est représentée sur la figure $2 G$ à $K$.

Dimensions : Mâle holotype : corps long de $8,3 \mathrm{~mm}$ et large de $45 \mu \mathrm{m}$; anneau nerveux à $165 \mu \mathrm{m}$ de l'apex; œsophage long de $360 \mu \mathrm{m}$; queue, spicule gauche et spicule droit longs de $10 \mu \mathrm{m}, 72 \mu \mathrm{m}$ et $50 \mu \mathrm{m}$; testicule à $450 \mu \mathrm{m}$ de l'apex.

Mâles paratypes: corps longs de 9,3, 10,15, 10,2 mm, larges de 56, 58 et $68 \mu \mathrm{m}$; anneau nerveux à 135, 132 et $140 \mu \mathrm{m}$ de l'apex; œsophages longs de 355, 280 et $400 \mathrm{l} \mu \mathrm{m}$; queues longues de 12,9 et $12 \mu \mathrm{m}$; spicules gauches longs de 68,70 et $65 \mu \mathrm{m}$ et spicules droits longs de 57,55 et $52 \mu \mathrm{m}$; testicules à 540,650 et $565 \mu \mathrm{m}$ de l'apex.

Microfilaires sanguines : immobilisées par la chaleur : corps longs de 110 à $122 \mu \mathrm{m}$ $(122,110,116,122,115,115,118$ et $117 \mu \mathrm{m})$, larges de 3,5 à $4 \mu \mathrm{m}$; corps interne de 60 à $68 \mu \mathrm{m}$ de l'apex et long de 4-5 $\mu \mathrm{m}$; 


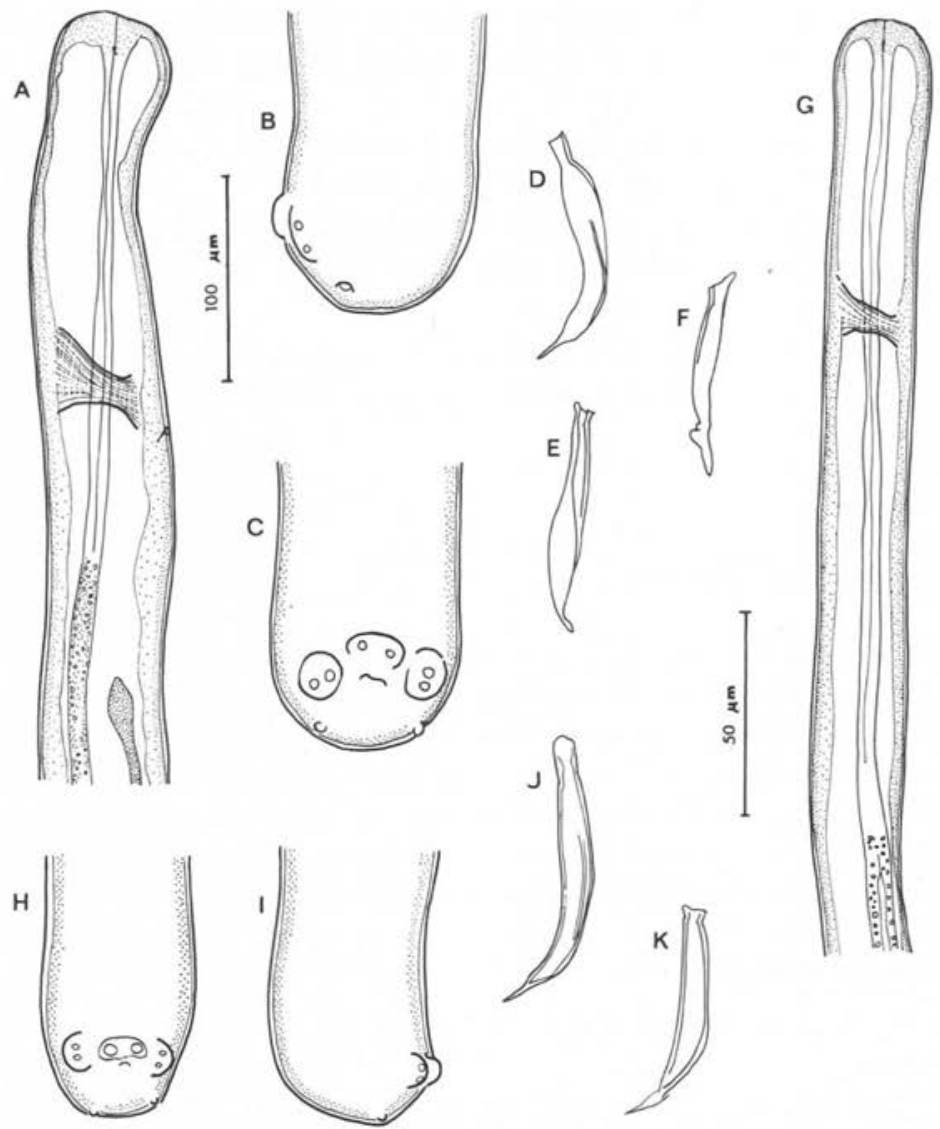

Fig. 2. A à $\mathrm{F}$ : Eufilaria delicata, mâle; $\mathrm{A}$ : région antérieure, vue latérale; $\mathrm{B}$ et $\mathrm{C}$ : région caudale, vues latérale et ventrale; D et $\mathrm{E}$ : spicule gauche, vues latérale gauche et ventrale; F : spicule droit, vue latérale gauche; G à $\mathrm{K}: E$. bartlettae n. sp., mâle; G : région antérieure ; $\mathrm{H}$ et $\mathrm{I}$ : région caudale, vues ventrale et latérale; $\mathrm{J}$ et $\mathrm{K}$ : spicules gauche et droit, vue latérale gauche. (A, G, éch. $100 \mu \mathrm{m}$; B, C, D, E, F, H, I, J, K, éch. $50 \mu \mathrm{m}$ ).

- colorées vitalement : corps long de $116 \mu \mathrm{m}$, large de $4 \mu \mathrm{m}$, anneau nerveux et noyau excréteur à $22 \mu \mathrm{m}$ et $43 \mu \mathrm{m}$ de l'apex; corps interne à $65 \mu \mathrm{m}$ de l'apex et long de $5 \mu \mathrm{m} ; \mathrm{R}_{1}, \mathrm{R}_{2}$ et $\mathrm{R}_{4}$ à 75,85 et $93 \mu \mathrm{m}$ de l'apex (fig. $3 \mathrm{D}$ ).

\section{Discussion.}

Le pluriparasitisme chez un hôte déterminé par microspéciations successives à partir d'une espèce est fréquemment observé chez les Nématodes intestinaux (Chabaud et Durette-Desset, 1978) et existe également chez les Filaires (ex. les Waltonella des Bufo marinus, cf. Bain, Kim et Petit, 1979). 
On sait, par ailleurs, que les phénomènes de spéciation fine chez les Filaires se manifestent avant tout par des différences morphologiques de la microfilaire et non des adultes.

Ces considérations font que nous devons attribuer une valeur réelle à de faibles différences morphologiques constatées chez les adultes.

Les quatre mâles du matériel 104 EJ s'opposent au mâle unique 105 EJ par le fait que les spicules gauche et droit sont un peu plus inégaux $(11 \mu \mathrm{m}$ à $22 \mu \mathrm{m}$ d'écart au lieu de $8 \mu \mathrm{m}$ ).

Nous sommes donc amenés à attribuer ces spécimens à la seconde espèce qui existe chez ces merles et les déterminons comme $E$. bartlettae n. sp., dédiée à notre collègue $\mathrm{C}$. Bartlett.

\section{III - Développement larvaire chez Culicoides nubeculosus}

La microfilarémie du Merle 105 EJ est de une à deux microfilaires/10 $\mu \mathrm{l}$ de sang dans la journée et de 15 à 22 microfilaires/10 $\mu$ l vers minuit. Les Culicoides sont donc gorgés au moment du pic nocturne. Trois séries de gorgement sont effectuées; les Culicoides sont maintenus à $26^{\circ}$ et disséqués à trois temps différents ; 48 femelles le quatrième jour: trois ont un stade 1 situé dans le thorax et un très jeune stade II ; 206 femelles le $7^{\mathrm{e}}$ jour : une a un stade subinfestant ; 230 femelles le $8^{\mathrm{e}}$ jour : une a deux stades infestants et quatre ont un stade infestant, tous situés dans la tête.

\section{A - Eufilaria delicata.}

Le cycle est connu par un stade I de 4 jours ( $f i g .3 B$ et $C$ ) et un stade infestant mâle de 8 jours.

Stade I : il est caractérisé par le bouchon anal bien saillant, la queue concave terminée par un assez long filament caudal. Corps long de $165 \mu \mathrm{m}$, large de $21 \mu \mathrm{m}$; anneau nerveux et pore excréteur à 25 et $42 \mu \mathrm{m}$ de l'apex; œsophage long de $75 \mu \mathrm{m}$; rectum long de $33 \mu \mathrm{m}$; queue longue de $35 \mu \mathrm{m}$ et filament caudal de $18 \mu \mathrm{m}$.

Stade infestant : corps long de $568 \mu \mathrm{m}$, large de $12 \mu \mathrm{m}$; anneau nerveux et pore excréteur à 62 et $100 \mu \mathrm{m}$ de l'apex; œsophage long de $360-440 \mu \mathrm{m}$ (le passage de l'œsophage à l'intestin se fait insensiblement) avec une portion musculaire de $140 \mu \mathrm{m}$; rectum et queue longs de 42 et $20 \mu \mathrm{m}$; ébauche génitale à $275 \mu \mathrm{m}$ de l'apex.

\section{B - Eufilaria bartlettae n. sp.}

Le cycle est connu par deux stades I, un stade II juste après la mue, ainsi que par cinq stades infestants; nous rattachons le stade subinfestant à cette espèce, qui est la plus fréquente (fig. $3 E$ à $K$ ).

Stade I : il a un bouchon cuticulaire anal plat, une queue non concave et une pointe caudale courte. Une larve est longue de $167 \mu \mathrm{m}$, large de $21 \mu \mathrm{m}$; anneau 


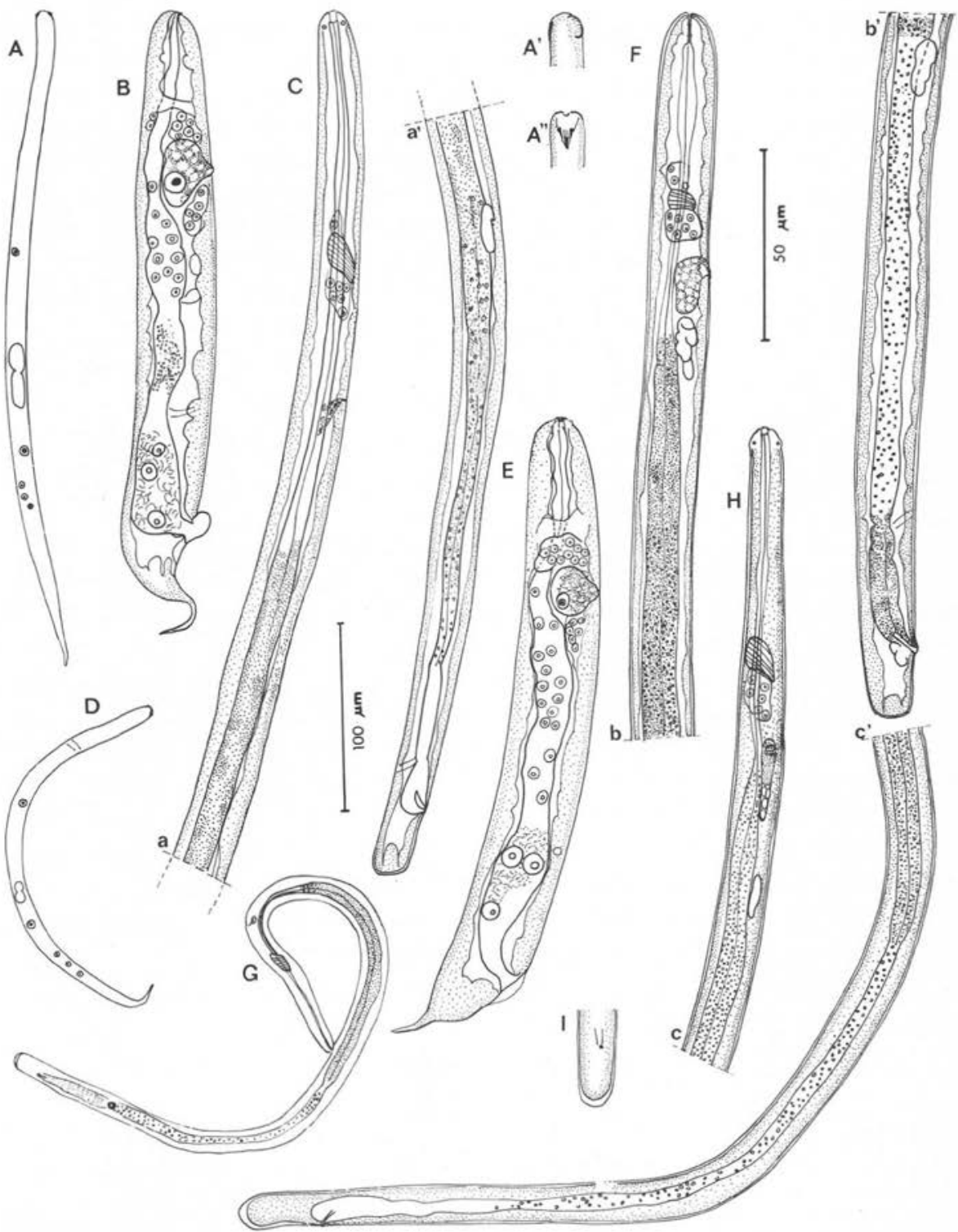

Fig. 3. $\mathrm{A}$ à $\mathrm{C}$ : Eufilaria delicata ; $\mathrm{A}$ : microfilaire sanguine colorée vitalement ; $\mathrm{A}^{\prime}$ et $\mathrm{A}^{\prime \prime}$ : idem, tête, crochet de profil et de face ; B : stade I de 4 jours, vue latérale; C: larve infestante mâle (les régions antérieure et postérieure sont représentées mais non la région moyenne); D à $\mathrm{K}:$ E. bartlettae n. sp.; D: microfilaire sanguine colorée vitalement; E : stade I de 4 jours, vue latérale; F: larve subinfestante entière, en deux morceaux $\left(b, b^{\prime}\right)$, vue latérale; $\mathrm{G}$ : larve infestante; $\mathrm{H}$ : autre larve infestante femelle, en deux morceaux (c, c'), vue latérale ; I : queue, vue ventrale. (A, B, C, D, E, F, G, H, I, éch. $50 \mu \mathrm{m}$; $\mathrm{A}^{\prime}, \mathrm{A}^{\prime \prime}$, à main levée). 
nerveux et pore excréteur à $28 \mu \mathrm{m}$ et $44 \mu \mathrm{m}$ de l'apex; œsophage long de $80 \mu \mathrm{m}$; rectum long de $42 \mu \mathrm{m}$; queue longue de $31 \mu \mathrm{m}$ et filament caudal de $10 \mu \mathrm{m}$.

Stade subinfestant : corps long de $370 \mu \mathrm{m}$, large de $16 \mu \mathrm{m}$; capsule buccale haute de $8 \mu \mathrm{m}$; anneau nerveux et pore excréteur à 45 et $62 \mu \mathrm{m}$ de l'apex; œsophage long de $180 \mu \mathrm{m}$ (portion musculaire de $70 \mu \mathrm{m}$ ) ; rectum et queue longs de $37 \mu \mathrm{m}$ et $19 \mu \mathrm{m}$.

Stades infestants : corps longs de 410, 445, 420, 410 et $485 \mu \mathrm{m}$, larges de 14, 12, 13,12 et $13 \mu \mathrm{m}$; anneaux nerveux à $50,53,55,58$ et $55 \mu \mathrm{m}$ de l'apex ; pore excréteur à $85,88,82,80$ et $76 \mu \mathrm{m}$ de l'apex; œsophages longs environ de 300, 315, 210, 210 et ? $\mu \mathrm{m}$ avec portions musculaires de $72,105,95,80$ et $95 \mu \mathrm{m}$; rectums longs de $36,42,48,45$ et $38 \mu \mathrm{m}$; queues longues de 15, 15, 16, 20 et $16 \mu \mathrm{m}$.

\section{Discussion}

Les trois cycles d'Eufilaria connus [E. longicaudata Hibler, 1964 (cf. Hibler, 1964), E. delicata et E. bartlettae n. sp.] s'effectuent chez des Culicoides et le développement a lieu dans les muscles thoraciques.

Les cycles évolutifs de Filaires parasites d'Oiseaux restent actuellement très peu connus; les morphologies larvaires n'ont été étudiées en détail que dans trois genres: Splendidofilaria Skrjabin, 1923 (cf. Anderson, 1956), Chandlerella Yorke \& Maplestone, 1926 (cf. Bartlett, 1979) et Eufilaria.

Il s'agit précisément de cycles évolutifs s'effectuant chez des Ceratopogonidés ou des Simulidés. Il est intéressant de constater que, dans l'état actuel de nos connaissances, les morphologies des stades larvaires, bien qu'extrêmement proches les unes des autres (larves infestantes de 0,4 à $0,6 \mathrm{~mm}$, avec séparation de l'œsophage et de l'intestin peu perceptible) peuvent être distinguées par les extrémités caudales :

- Splendidofilaria fallisensis (Anderson, 1954): stade I avec une petite pointe caudale, stade infestant avec deux languettes latérales subsphériques.

- Chandlerella chitwoodae Anderson, 1961: stade I à extrémité caudale arrondie, stade infestant à extrémité conique, infléchie ventralement.

- Eufilaria spp. (cf. ci-dessus). Stade I à pointe caudale longue, stade infestant à queue tronquée ou arrondie.

\section{Bibliographie}

Anderson R.C.: The life cycle and seasonal transmission of Ornithofilaria fallisensis Anderson, a parasite of domestic and wild ducks. Canad. J. Zool., 1956, 34, 485-525.

Bain O., Kim D.-C., Petit G.: Diversité spécifique des Filaires du genre Waltonella coexistant chez Bufo marinus. Bull. Mus. Nat. Hist. Nat. Paris, 1979, $4^{e}$ sér., 1, sect. A, 199-212.

Bartlett C. M. : Filarioid nematodes (Filarioidea : Onchocercidae) of the crow (Corvus brachyrhynchos brachyrhynchos Brehm) in Southern Ontario. Thèse, Master of Science, Univ. Guelph., 1979 155 pp. polycopiées.

Chabaud A.-G., Durette-Desset M.-C.: Parasitisme par plusieurs espèces congénériques. Bull. Soc. Zool. France, 1978, 103, 459-464.

Hibler C. P.: Onchocercidae (Nematoda : Filarioidea) of the American magpie Pica pica hudsonia (Sabine) in Northern Colorado. Dissert. Abst., 1964, 24, 4323-4324.

Supperer R. : Zwei neue Filarien (S.L.) Eufilaria delicata spec. nov. und Ornithofilaria böhmi spec. nov. aus der Misteldrossel, Turdus viscivorus L. Z. f. Parasitenkunde, 1958, 18, 312-319. 